\title{
THE KHINCHIN INEQUALITY AND CHEN'S THEOREM
}

\author{
M. M. SKRIGANOV \\ To Vasiliu Mikhaũlovich Babich, on the occasion of his 80th birthday, \\ with unwavering admiration
}

\begin{abstract}
Chen's theorem on the mean values of $L_{q}$-discrepancies is one of the basic results in the theory of uniformly distributed point sets. This is a difficult result, based on deep and nontrivial combinatorial arguments (see the papers by Chen and Beck on irregularities of distributions). The paper is aimed at showing that the results of such a type are intimately related to lacunarity and statistical independence of certain function series. In particular, the classical Khinchin inequality for the Rademacher functions is employed to prove an important generalization of Chen's theorem. In a forthcoming paper, the author will continue the study of the phenomena of lacunarity and statistical independence in the context of the theory of uniformly distributed point sets.
\end{abstract}

\section{$\S 1$ Introduction. The main theorem}

1.1. Let $D_{N}$ be an arbitrary subset (distribution) of $N>1$ points in the $d$-dimensional unit cube $U^{d}=[0,1)^{d}$. Define the local discrepancy $\mathcal{L}\left[D_{N}, Y\right], Y=\left(y_{1}, \ldots, y_{d}\right) \in U^{d}$, as

$$
\mathcal{L}\left[D_{N}, Y\right]=\#\left\{D_{N} \cap B_{Y}\right\}-N \operatorname{vol} B_{Y},
$$

where $B_{Y}=\left[0, y_{1}\right) \times \cdots \times\left[0, y_{d}\right) \subset U^{d}$ is a rectangular box of volume vol $B_{Y}=y_{1} \ldots y_{d}$, and the $L_{q}$-discrepancy $\mathcal{L}_{q}\left[D_{N}\right], 1 \leq q<\infty$, as

$$
\mathcal{L}_{q}\left[D_{N}\right]=\left(\int_{U^{d}}\left|\mathcal{L}\left[D_{N}, Y\right]\right|^{q} d Y\right)^{1 / q} .
$$

It is known that, for $1<q<\infty$ and for an arbitrary distribution $D_{N}$, we have the lower bound

$$
\mathcal{L}_{q}\left[D_{N}\right]>c_{d, q}(\log N)^{\frac{1}{2}(d-1)}
$$

with a constant $c_{d, q}$ independent of $N$. This is a theorem of Roth for $q \geq 2$ and a theorem of Schmidt for $1<q<2$ (see [1, 7]).

The order of the bound (1.3) is the best possible. Namely, for each $q, 1 \leq q<\infty$, and any $N>1$ there exist distributions $D_{N}$ of $N$ points in $U^{d}$ such that

$$
\mathcal{L}_{q}\left[D_{N}\right]<C_{d, q}(\log N)^{\frac{1}{2}(d-1)}
$$

with a constant $C_{d, q}$, independent of $N$. For $1 \leq q \leq 2$ this result was obtained by Davenport for $d=2$, by Roth for $d \geq 3$, and by Chen for arbitrary $q$ and $d$ (see [1, 2]).

Chen's proof is based on a mean-value theorem for the $L_{q}$-discrepancies with respect to the $p$-adic shifts of a given distribution $D_{N}$. This very important theorem was proved in [2] with the help of deep and nontrivial combinatorial arguments.

2010 Mathematics Subject Classification. Primary 11K36.

Key words and phrases. Uniform distributions, harmonic analysis, lacunary series.

Partially supported by RFBR (project no. 08-01-00182). 
In the present paper we propose a radically new approach to the proof of meanvalue theorems for $L_{q}$-discrepancies; this approach is based on the methods of harmonic analysis. With the help of this approach, we shall give an essential improvement of Chen's theorem.

1.2. We recall some definitions and well-known facts.

We write $\mathbb{N}$ for the set of all positive integers, $\mathbb{N}_{0}$ for the set of all nonnegative integers, and $\mathbb{N}^{d}, \mathbb{N}_{0}^{d}$ for the products of $d$ copies of the corresponding sets. For $s \in \mathbb{N}_{0}$, we put

$$
\begin{aligned}
\mathbb{Q}\left(2^{s}\right) & =\left\{x=m 2^{-s} \in[0,1): m=0,1, \ldots, 2^{s}-1\right\}, \\
\mathbb{Q}^{d}\left(2^{s}\right) & =\left\{x=\left(x_{1}, \ldots, x_{d}\right) \in U^{d}: x_{j} \in \mathbb{Q}\left(2^{s}\right), j=1, \ldots, d\right\}, \\
\mathbb{Q}\left(2^{\infty}\right) & =\bigcup_{s \geq 0} \mathbb{Q}\left(2^{s}\right), \quad \mathbb{Q}^{d}\left(2^{\infty}\right)=\bigcup_{s \geq 0} \mathbb{Q}^{d}\left(2^{s}\right) .
\end{aligned}
$$

The points of $\mathbb{Q}^{d}\left(2^{\infty}\right)$ are called dyadic rational points.

Any $x \in[0,1)$ can be represented in the form

$$
x=\sum_{i \geq 1} \eta_{i}(x) 2^{-i}
$$

where $\eta_{i}(x) \in\{0,1\} \simeq \mathbb{F}_{2}, i \in \mathbb{N}$. Here $\mathbb{F}_{2}$ is the field of two elements identified with the set of residues $\{0,1\} \bmod 2$.

The dyadic expansion (1.6) is unique if we agree that for each dyadic rational point the sum in (1.6) contains finitely many nonzero terms. With this agreement, $\eta_{i}(x)=0$ for $i>s$ if $x \in \mathbb{Q}\left(2^{s}\right)$.

On the set of dyadic rational points, the structure of a vector space over the finite field $\mathbb{F}_{2}$ can be defined naturally. For any two points $x$ and $y$ in $\mathbb{Q}\left(2^{\infty}\right)$, we define their sum $x \oplus y$ as follows:

$$
\eta_{i}(x \oplus y)=\eta_{i}(x)+\eta_{i}(y) \bmod 2, \quad i \in \mathbb{N} .
$$

Similarly, for any two vectors $X=\left(x_{1}, \ldots, x_{d}\right)$ and $Y=\left(y_{1}, \ldots, y_{d}\right)$ in $\mathbb{Q}^{d}\left(2^{\infty}\right)$ we define

$$
X \oplus Y=\left(x_{1} \oplus y_{1}, \ldots, x_{d} \oplus y_{d}\right) .
$$

With respect to the addition $\oplus$ defined in this way, each set $\mathbb{Q}^{d}\left(2^{s}\right)$ is a vector space over the field $\mathbb{F}_{2}$, and $\operatorname{dim} \mathbb{Q}^{d}\left(2^{s}\right)=d s, \#\left\{\mathbb{Q}^{d}\left(2^{s}\right)\right\}=2^{d s}$.

For an arbitrary point $x \in[0,1)$ with dyadic expansion $(1.6)$, we denote by $x^{(s)}$ its projection to $\mathbb{Q}\left(2^{s}\right)$ :

$$
x^{(s)}=\sum_{i=1}^{s} \eta_{i}(x) 2^{-i}, \quad s \in \mathbb{N},
$$

and for $s=0$ we put $x^{(0)}=0$, so that

$$
x=x^{(s)}+\theta_{s}(x) 2^{-s}, \quad s \in \mathbb{N}_{0},
$$

where $\theta_{s}(x) \in[0,1)$ for all $x \in[0,1)$.

Similarly, for a point $X=\left(x_{1}, \ldots, x_{d}\right) \in U^{d}$ we denote by $X^{(s)}=\left(x_{1}^{(s)}, \ldots, x_{d}^{(s)}\right)$ its projection to $\mathbb{Q}^{d}\left(2^{s}\right)$, so that

$$
X=X^{(s)}+\Theta_{s}(X) 2^{-s}, \quad s \in \mathbb{N}_{0},
$$

where $\Theta_{s}(X)=\left(\theta_{s}\left(x_{1}\right), \ldots, \theta_{s}\left(x_{d}\right)\right) \in U_{d}$ for all $X \in U^{d}$. For $X \in \mathbb{Q}^{d}\left(2^{s}\right)$, "the error" $\Theta_{s}(X)$ is 0 .

Notice that the addition $\oplus$ can be consistently defined for pairs of points $X, Y$ if only one of the points, say $Y$, belongs to $\mathbb{Q}^{d}\left(2^{s}\right)$. For $X \in U^{d}$ and $Y \in \mathbb{Q}^{d}\left(2^{s}\right)$, we put

$$
X \oplus Y=X^{(s)} \oplus Y+\Theta_{s}(x) 2^{-s} .
$$


For $x \in[0,1)$ and $y \in \mathbb{Q}\left(2^{s}\right)$, formula (1.12) can be written in the following form:

$$
\eta_{i}(x \oplus y)= \begin{cases}\eta_{i}(x)+\eta_{i}(y) \bmod 2 & \text { if } 1 \leq i \leq s, \\ \eta_{i}(x) & \text { if } i>s .\end{cases}
$$

These subtle questions were discussed in [5] and [6] in more detail.

What has been said above shows that, for an arbitrary distribution of $N$ points $D_{N} \subset$ $U^{d}$ and any point $T \in \mathbb{Q}^{d}\left(2^{s}\right)$, we can define the dyadic shift $D_{N} \oplus T=\{X \oplus T: X \in$ $\left.D_{N}\right\} \subset U^{d}$ and view it as a new distribution of $N$ points in $Y^{d}$.

1.3. Following Chen [1, 2, for a distribution $D$ we define the following mean values for the $L_{q}$-discrepancies:

$$
\mathcal{M}_{s, q}[D]=\left(2^{-d s} \sum_{T \in \mathbb{Q}^{d}\left(2^{s}\right)}\left(\mathcal{L}_{q}\left[D_{N} \oplus T\right]\right)^{q}\right)^{1 / q} .
$$

Our aim is to study the means (1.14) for some special class of point distributions. Consider the elementary intervals $\Delta_{a}^{m} \subset[0,1)$, where $a, m \in \mathbb{N}_{0}$ :

$$
\Delta_{a}^{m}=\left[m 2^{-a},(m+1) 2^{-a}\right), \quad 0 \leq m<2^{a},
$$

and the elementary boxes $\Delta_{A}^{M} \subset U^{d}$, where $A=\left(a_{1}, \ldots, a_{d}\right), M=\left(m_{1}, \ldots, m_{d}\right) \in \mathbb{N}_{0}^{d}$ :

$$
\Delta_{A}^{M}=\Delta_{a}^{m_{1}} \times \cdots \times \Delta_{a_{d}}^{m_{d}}, \quad 0 \leq m_{j}<2^{a_{j}}, j=1, \ldots, d .
$$

Every such box has volume vol $\Delta_{A}^{M}=2^{-a_{1}-\cdots-a_{d}}$.

Recall the following definition. Let $0 \leq \delta \leq s$ be integers. A subset $D \subset U^{d}$ consisting of $N=2^{s}$ points is called a dyadic $(\delta, s, \bar{d})$-net of deficiency $\delta$ if each elementary box $\Delta_{A}^{M}$ of volume $2^{\delta-s}$ contains exactly $2^{\delta}$ points of $D$.

Notice that for any $(\delta, s, d)$-net $D$ and any $T \in \mathbb{Q}^{d}\left(2^{\infty}\right)$ the shift $D \oplus T$ is also a net with the same parameters.

The $(\delta, s, d)$-nets are very interesting combinatorial structures. The first example of a $(0, s, 2)$-net, $s \in \mathbb{N}_{0}$, was constructed by Van der Corput (see [1]). In arbitrary dimensions $d$, first constructions of dyadic $(\delta, s, d)$-nets with $\delta=O(d \log d)$ were given by $\operatorname{Sobol}^{\prime}[12$.

Later, other constructions of nets were also proposed. Replacing the base 2 in the definition and in all formulas (1.9)-(1.16) by an arbitrary prime base $p$, we arrive at $(\delta, s, d)$-nets in base $p$, which also have many remarkable properties (see [1, 4]). By using the methods of algebraic geometry over finite fields, the existence of $(\delta, s, d)$-nets with $\delta=O(d)$ can be proved, and this bound is already the best possible for large $d$ (see [8]). It is significant that $(0, s, d)$-nets in a prime base $p$ and with arbitrarily large $s$ exist if and only if $d \leq p+1$. In particular, infinite sequences of dyadic nets with $\delta=0$ exist only in dimensions $d=1,2$, and 3 (see [1, 11, 12]).

$(\delta, s, d)$-nets fill the unit cube very uniformly, and for all $q$ their $L_{q}$-discrepancies admit a bound of the order of $O\left(2^{\delta} s^{d-1}\right)$ as $s \rightarrow \infty$. For arbitrary $(\delta, s, d)$-nets, in general, this bound is best possible. At the same time, this bound is still very far from the lower bound (1.3).

The following question arises naturally: in arbitrary dimensions, do dyadic $(\delta, s, d)$ nets exist that satisfy (1.3)? An affirmative answer to this question follows at once from the main theorem of the present paper.

Theorem 1.1. If $D \subset U^{d}$ is a dyadic $(\delta, s, d)$-net, then for any $q \geq 2$ the mean value of the $L_{q}$-discrepancies (1.14) satisfies the estimate

$$
\mathcal{M}_{s, q}[D]<\gamma(q, \delta, d)(s+1)^{\frac{1}{2}(d-1)},
$$


where we can put

$$
\gamma(q, \delta, d)=4^{\delta+d+1} q^{\frac{1}{2}(d+1)} .
$$

For the first time, bounds (1.17) were given by Chen (see [1, 2]) for nets of deficiency $\delta=0$ on the basis of an elaborate combinatorial analysis involving simultaneous induction on the parameters $d, s$, and even integers $q$. For this approach, the assumption $\delta=0$ turns out to be essential; as a result, for each fixed prime base $p$ Chen's theorem can be applied only to dimensions $d$ not exceeding $p+1$, and for dyadic nets only to dimensions 1 , 2 , and 3.

In the present paper we develop a new approach to the study of the mean value of the $L_{q}$-discrepancies (1.14); under this approach, the value $\delta$ of the deficiency of the net turns out to be completely irrelevant. Our approach is based on the theory of lacunary series of functions. In the case of dyadic $(\delta, s, d)$-nets, these are series of Rademacher functions, which form a lacunary subsystem for the Walsh functions, and in the case of nets in an arbitrary base $p$ these series are lacunary subsystems for the corresponding Chrestenson-Levy functions (see [6, 11]).

In the present publication we do not aim at presenting our results in the most general form. Instead, here we concentrate on the main ideas of our approach. For this reason, in Theorem 1.1 we consider only dyadic $(\delta, s, d)$-nets. In this case, to prove the bound $(1.17)$ we need only the classical Khinchin inequality for the Rademacher functions.

For fixed $d, \delta$, and $q$, the bound (1.17) is best possible as $s \rightarrow \infty$, but the constant (1.18) can be improved substantially and replaced by

$$
\gamma(q, \delta, d)=c_{d} 2^{\delta} q^{\frac{1}{2}(d-1)}
$$

with a constant $c_{d}$ depending on dimension only. The author hopes to consider these questions in forthcoming publications.

The paper is organized as follows. In $\S 2$, the necessary facts about Walsh and Rademacher functions are listed and the Kinchin inequality is given in the form convenient for our purposes. In $\S 3$ we obtain explicit formulas for discrepancies and introduce the concept of disbalance, which is an important characteristic of a point distribution. In Theorem 3.1, we use Khinchin's inequality to estimate the mean values of discrepancies for arbitrary distributions in terms of their disbalances. In $\S 4$, in Lemma 4.1 we show that the $(\delta, s, d)$-nets are well-balanced distributions because their disbalances are small. Using Theorem 3.1 and Lemma 4.1, we complete the proof of Theorem 1.1 in $\S 4$.

\section{§2. The Walsh and Rademacher functions. Khinchin's inequality}

2.1. The Walsh functions would be properly thought of as characters of a totally disconnected topological group, the so-called dyadic Cantor group (see [5, 6, 11]). However, such an advanced theory is not needed for the purposes of the present paper. Instead of this, we shall consider the finite groups $\mathbb{Q}\left(2^{s}\right)$, which can be thought of as appropriate approximations to Cantor's group (see [11]).

Any integer $l \in \mathbb{N}_{0}$ can be represented uniquely in the form

$$
\ell=\sum_{i \geq 1} \lambda_{i}(\ell) 2^{i-1}
$$

where $\lambda_{i}(\ell) \in\{0,1\} \simeq \mathbb{F}_{2}, i \in \mathbb{N}$. On the set $\mathbb{N}_{0}$, the structure of a vector space over the finite field $\mathbb{F}_{2}$ can be defined naturally. For any two integers $\ell$ and $k$ in $\mathbb{N}_{0}$, we define their sum $\ell \oplus k$ by the formula

$$
\lambda_{i}(\ell \oplus k)=\lambda_{i}(\ell)+\lambda_{i}(k) \bmod 2, \quad i \in \mathbb{N} .
$$


Similarly, for any two vectors $L=\left(\ell_{1}, \ldots, \ell_{d}\right)$ and $K=\left(k_{1}, \ldots, k_{d}\right)$ in $\mathbb{N}_{0}^{d}$, let

$$
L \oplus K=\left(\ell_{1} \oplus k_{1}, \ldots, l_{d} \oplus k_{d}\right) .
$$

It is convenient to introduce the following notation. For $s \in \mathbb{N}_{0}$ we put

$$
\mathbb{N}_{0}\left(2^{s}\right)=\left\{\ell \in \mathbb{N}_{0}: 0 \leq l<2^{s}\right\}
$$

and

$$
\mathbb{N}_{0}^{d}\left(2^{s}\right)=\left\{L=\left(\ell_{1}, \ldots, \ell_{d}\right) \in \mathbb{N}_{0}^{d}: \ell_{j} \in \mathbb{N}_{0}\left(2^{s}\right), j=1, \ldots, d\right\},
$$

and for $A=\left(a_{1}, \ldots, a_{d}\right) \in \mathbb{N}_{0}^{d}$ we put

$$
\mathbb{N}_{0}^{d}\left(2^{A}\right)=\left\{L=\left(\ell_{1}, \ldots, \ell_{d}\right) \in \mathbb{N}_{0}^{d}: \ell_{j} \in \mathbb{N}_{0}\left(2^{a_{j}}\right), j=1, \ldots, d\right\} .
$$

With respect to the addition defined in this way, each set $\mathbb{N}_{0}^{d}\left(2^{A}\right)$ is a vector space over the field $\mathbb{F}_{2}$, and $\operatorname{dim} \mathbb{N}_{0}\left(2^{A}\right)=a_{1}+\cdots+a_{d}$, and $\#\left\{\mathbb{N}_{0}^{d}\left(2^{A}\right)\right\}=2^{a_{1}+\cdots+a_{d}}$.

For any $\ell \in \mathbb{N}_{0}$ and $x \in[0,1)$ we define the pairing

$$
\langle\ell, x\rangle=\sum_{i \geq 1} \lambda_{i}(\ell) \eta_{i}(x) \bmod 2,
$$

where the $\lambda_{i}(\ell)$ and $\eta_{i}(x)$ are the coefficients in the expansions (2.1) and (1.9), respectively. For $L=\left(\ell_{1}, \ldots, \ell_{d}\right) \in \mathbb{N}_{0}^{d}$ and $X=\left(x_{1}, \ldots, x_{d}\right) \in U^{d}$, put

$$
\langle L, X\rangle=\sum_{j=1}^{d}\left\langle\ell_{j}, x_{j}\right\rangle \bmod 2 .
$$

The Walsh functions in $d$ dimensions are defined by

$$
W_{L}(X)=(-1)^{\langle L, X\rangle} \text {. }
$$

Obviously,

$$
W_{L}(X)=\prod_{j=1}^{d} w_{\ell_{j}}\left(x_{j}\right),
$$

where the $w_{\ell}(x)$ are the one-dimensional Walsh functions

$$
w_{\ell}(x)=(-1)^{\sum_{i \geq 1} \lambda_{i}(\ell) \eta_{i}(x)} .
$$

The Walsh functions $w_{\ell}(x), \ell \in \mathbb{N}$, take only two values \pm 1 . Furthermore, $w_{\ell}(x), \ell \in$ $\mathbb{N}_{0}$, is piecewise constant on all elementary intervals $\Delta_{a}^{m}=\left[m 2^{-a},(m+1) 2^{-a}\right) \subset[0,1)$, $0 \leq m<2^{a}$, provided that $a \geq \rho(\ell)$, where

$$
\rho(\ell)= \begin{cases}0 & \text { if } \ell=0, \\ \max \left\{i: \lambda_{i}(\ell) \neq 0\right\} & \text { if } \ell \in \mathbb{N},\end{cases}
$$

and for $a<\rho(\ell)$ we have

$$
\int_{\Delta_{a}^{m}} w_{\ell}(x) d x=0
$$

Next, for $\ell \in \mathbb{N}_{0}\left(2^{s}\right)$ we have

$$
w_{\ell}(x)=w_{\ell}\left(x^{(s)}\right),
$$

where $x^{(s)}$ is the projection of a point $x \in[0,1)$ to $\mathbb{Q}\left(2^{s}\right)$ (see (1.9)).

In terms of the function $\rho(\ell)$, the elementary intervals (1.15) and the boxes (1.16) can be written in the form

$$
\begin{aligned}
\mathbb{N}_{0}\left(2^{s}\right) & =\{\ell \in \mathbb{N}: \rho(\ell) \leq s\} \\
\mathbb{N}_{0}\left(2^{A}\right) & =\left\{L=\left(\ell_{1}, \ldots, \ell_{d}\right) \in \mathbb{N}_{0}^{d}: \rho\left(\ell_{j}\right) \leq a_{j}, j=1, \ldots, d\right\}
\end{aligned}
$$


It should be noted that on the set of integers $\mathbb{N}_{0}$ with the addition $\oplus$ the expression $\rho(\ell \oplus k)$ defines the Rosenbloom-Tsfasman metric. This (non-Archimedean) metric turns out to be intimately related to the geometric structure of uniformly distributed point sets (see [10, 11]). However, in the present paper we do not dwell on these questions, using (2.11) merely as a convenient notation.

For all $L, K \in \mathbb{N}_{0}^{d}$ and $X \in U^{d}$ we have

$$
W_{L \oplus K}(x)=W_{L}(x) W_{K}(x),
$$

and also

$$
W_{L}(X \oplus Y)=W_{L}(X) W_{L}(Y)
$$

provided that for $X, Y \in U^{d}$ their sum $X \oplus Y$ is well defined (e.g., if $Y \in \mathbb{Q}^{d}\left(2^{\infty}\right)$ ).

In particular, for each $s \in \mathbb{N}_{0}$ the Walsh functions $W_{L}(X), L \in \mathbb{N}_{0}^{d}\left(2^{s}\right)$, restricted to $X \in \mathbb{Q}^{d}\left(2^{s}\right)$ form a group of all characters of the additive group of the vector space $\mathbb{Q}^{d}\left(2^{s}\right)$. This implies the relations

$$
2^{-d s} \sum_{X \in \mathbb{Q}^{d}\left(2^{s}\right)} W_{L}(X)= \begin{cases}1 & \text { if } L=0 \\ 0 & \text { if } L \in \mathbb{N}_{0}^{d}\left(2^{s}\right) \backslash\{0\} .\end{cases}
$$

The Walsh functions form a complete orthonormal system in $L_{2}\left(U^{d}\right)$ :

$$
\int_{U^{d}} W_{K}(X) W_{L}(X) d x=\int_{U^{d}} W_{K \oplus L}(X) d x=\delta(K \oplus L),
$$

where the Kronecker "delta" $\delta(k)$ is 0 if $k \in \mathbb{N}_{0}^{d} \backslash\{0\}$, and $\delta(0)=1$, and for any function $f \in L_{2}\left(U^{d}\right)$ we have the Fourier-Walsh expansion

$$
f(X) \simeq \sum_{L \in \mathbb{N}_{0}^{d}} \widehat{f}_{L} W_{L}(X),
$$

where the $\widehat{f}_{L}$ are the Fourier-Walsh coefficients

$$
\widehat{f}_{L}=\int_{U^{d}} W_{L}(X) f(X) d x,
$$

and the symbol $\simeq$ means either $L_{2}$-convergence, or merely the list of the Fourier-Walsh coefficients for a given function $f$. Since the Walsh functions are real-valued, we shall assume that all functions we deal with, as well as all coefficients in the Fourier-Walsh series are also real-valued.

Since the system of Walsh functions is complete and orthonormal, we have

$$
\|f\|_{2}=\sum_{L \in \mathbb{N}_{0}^{d}} \widehat{f}_{L}^{2}
$$

where, as usual, $\|\cdot\|_{q}$ denotes the standard norm in the space $L_{q}\left(U^{d}\right)$,

$$
\|f\|_{q}=\left(\int_{U^{d}}|f(X)|^{q} d x\right)^{1 / q}, \quad 1 \leq q<\infty .
$$

To handle Fourier-Walsh series pointwise, it is convenient to introduce piecewise constant approximations. For $s \in \mathbb{N}_{0}$, we write $\mathfrak{M}_{s}$ for the family of elementary cubes (see (1.15), (1.16)) of the form

$$
\Delta_{S}^{M}=\left[m_{1}, 2^{-s},\left(m_{1}+1\right) 2^{-s}\right) \times \cdots \times\left[m_{d} 2^{-s},\left(m_{d}+1\right) 2^{-s}\right),
$$


where $S=(s, \ldots, s)$ and $M=\left(m_{1}, \ldots, m_{d}\right) \in \mathbb{N}_{0}^{d}\left(2^{S}\right)$. Every such cube has volume $2^{-d s}$, and the family $\mathfrak{M}_{s}$ forms a partition of the unit cube $U^{d}$. For $f \in L_{1}\left(U^{d}\right)$, a piecewise constant approximation $f^{(s)}$ is defined by

$$
f^{(s)}(X)=2^{-d s} \int_{\Delta_{S}^{M}} f(Y) d Y \quad \text { for } \quad X \in \Delta_{S}^{M} \in \mathfrak{M}_{s} .
$$

Formula (2.24) immediately implies the following bound:

$$
\inf _{Y \in \Delta_{S}^{M}} f(Y) \leq f^{(s)}(X) \leq \sup _{Y \in \Delta_{S}^{M}} f(Y), \quad X \in \Delta_{S}^{M} .
$$

Some other simple properties of these approximations are listed below. They can be derived easily from the definitions and properties of Walsh functions given above. We refer the reader to 6 for more details.

Lemma 2.1. Let $f \in L_{1}\left(U^{d}\right)$ have the Fourier-Walsh series (2.19) and a piecewise constant approximations $f^{(s)}, s \in \mathbb{N}_{0}$. Then, for each $s$, the following statements are true.

(i) The Fourier-Walsh series $f^{(s)}$ is finite and has the form

$$
f^{(s)}(X)=\sum_{L \in \mathbb{N}_{0}^{d}\left(2^{s}\right)} \widehat{f}_{L} W_{L}(X)
$$

with the same Fourier-Walsh coefficients $f_{L}$ as in (2.19).

(ii) For each point $X \in U^{d}$,

$$
f^{(s)}(X)=f^{(s)}\left(X^{(s)}\right)
$$

where $X^{(s)}$ is the projection of $X$ to $\mathbb{Q}^{d}\left(2^{s}\right)$ (see (1.10), (1.11)).

(iii) We have

$$
2^{-d s} \sum_{X \in \mathbb{Q}^{d}\left(2^{s}\right)} f^{(s)}(X)=\int_{U^{d}} f^{(s)}(X) d x=\widehat{f_{0}} .
$$

2.2. In the one-dimensional case, the Rademacher functions $r_{i}(x), x \in[0,1), i \in \mathbb{N}$, can be defined by one of the following expressions:

$$
r_{i}(x)=w_{2^{i-1}}(x)=(-1)^{\eta_{i}(x)}=1-2 \eta_{i}(x),
$$

where the $\eta_{i}(x)$ are the coefficients in the dyadic expansion (1.6). It is convenient to put $r_{0}(x) \equiv 1$. Each Walsh function $w_{\ell}(x)$ can be written uniquely as a product of Rademacher functions:

$$
w_{\ell}(x)=\prod_{i \geq 1}\left(r_{i}(x)\right)^{\lambda_{i}(\ell)},
$$

where the $\lambda_{i}(\ell)$ are coefficients in the dyadic expansion (2.1).

The multidimensional Rademacher functions $R_{L}(X), L=\left(\ell_{1}, \ldots, \ell_{d}\right) \in \mathbb{N}_{0}^{d}, X=$ $\left(x_{1}, \ldots, x_{d}\right) \in U^{d}$, are defined by

$$
R_{L}(X)=\prod_{j=1}^{d} r_{\ell_{j}}\left(x_{j}\right) .
$$

It is clear that

$$
R_{L}(X \oplus Y)=R_{L}(X) R_{L}(Y)
$$

if the sum $X \oplus Y$ is well defined, e.g., if $Y \in \mathbb{Q}^{d}\left(2^{\infty}\right)$. 
Now we wish to give some simple and useful formulas for Walsh and Rademacher functions. Here it is convenient to assume the functions $w_{\ell}(x)$ and $r_{i}(x)$ to be continued periodically to the entire real axis. In this case,

$$
r_{i}(x)=r_{1}\left(2^{i-1} x\right), \quad x \in \mathbb{R}, \quad i \in \mathbb{N} .
$$

Comparing (2.26) and (1.6), we get

$$
\{x\}=\frac{1}{2}-\sum_{i \geq 1} 2^{-i-1} r_{i}(x),
$$

where $\{x\}$ is the fractional part of $x \in \mathbb{R}$.

Using (2.27), one can easily obtain the identity

$$
\sum_{\ell \in \mathbb{N}_{0}\left(2^{k}\right)} w_{\ell}(x)=\prod_{i=1}^{k}\left(1+r_{i}(x)\right), \quad k \in \mathbb{N} .
$$

We shall use the notation $\chi(\mathcal{E},$.$) for the characteristic function of a subset \mathcal{E} \subset U^{d}$,

$$
\chi(\mathcal{E}, X)= \begin{cases}1 & \text { if } X \in \mathcal{E}, \\ 0 & \text { if } \notin \mathcal{E} .\end{cases}
$$

Identity $(2.32)$ can be written in terms of the characteristic function $\chi\left(\Delta_{k}^{0},.\right)$ of an elementary interval $\Delta_{a}^{0} \subset[0,1)($ see $(1.15))$ :

$$
2^{-k} \sum_{\ell \in \mathbb{N}_{0}\left(2^{k}\right)} w_{\ell}(x)=\chi\left(\Delta_{k}^{0}, x\right), \quad k \in \mathbb{N}_{0} .
$$

In this form, (2.34) holds true also for $k=0$.

We introduce the following intervals in $\mathbb{N}_{0}$ :

$$
\Pi_{0}=\{0\}, \quad \Pi_{k}=\left\{\ell \in \mathbb{N}: 2^{k-1} \leq \ell<2^{k}\right\}, \quad k \in \mathbb{N} .
$$

Note that, by (2.11), the intervals $(2.35)$ can be defined as follows:

$$
\Pi_{k}=\left\{\ell \in \mathbb{N}_{0}: \rho(\ell)=k\right\}, \quad k \in \mathbb{N}_{0} .
$$

It is easy to evaluate a sum of Walsh functions over the intervals (2.35). Using (2.15) and (2.26), we get

$$
\sum_{\ell \in \Pi_{k}} w_{\ell}(x)=r_{k}(x) \sum_{a \in \mathbb{N}_{0}\left(2^{k-1}\right)} w_{a}(x), \quad k \in \mathbb{N} .
$$

Formulas (2.37) and (2.34) show that

$$
2^{-k-1} \sum_{\ell \in \Pi_{k}} w_{\ell}(x)=r_{k}(x) \chi\left(\Delta_{k-1}^{0}, x\right), \quad k \in \mathbb{N} .
$$

We put

$$
k^{*}= \begin{cases}0 & \text { if } k=0 \\ k-1 & \text { if } k \in \mathbb{N} .\end{cases}
$$

Identity (2.38) can be written in the form

$$
2^{-k^{*}} \sum_{\ell \in \Pi_{k}} w_{\ell}(x)=r_{k}(x) \chi\left(\Delta_{K^{*}}^{0}, x\right), \quad k \in \mathbb{N}_{0} .
$$

In this form, (2.40) holds true also for $k=0$.

Consider the following rectangular boxes $\Pi_{K} \subset \mathbb{N}_{0}^{d}, K=\left(k_{1}, \ldots, k_{d}\right) \in \mathbb{N}_{0}^{d}$ :

$$
\Pi_{K}=\Pi_{k_{1}} \times \cdots \times \Pi_{k_{d}}=\left\{L=\left(\ell_{1}, \ldots, \ell_{d}\right) \in \mathbb{N}_{0}^{d}: \rho\left(\ell_{j}\right) \leq k_{j}, \quad j=1, \ldots, d\right\},
$$


where the $\Pi_{k} \subset \mathbb{N}_{0}$ are the intervals (2.35), (2.36). We denote $K^{*}=\left(k_{1}^{*}, \ldots, k_{d}^{*}\right)$, where the $k_{j}^{*}, j=1, \ldots, d$, are as in $(2.39)$.

Multiplying formulas (2.34) with $k=k_{j}, j=1, \ldots, d$, and doing similarly with formulas $(2.40)$ for $k=k_{j}, j=1, \ldots, d$, we arrive at the following statement.

Lemma 2.2. For each $k=\left(k_{1}, \ldots, k_{d}\right) \in \mathbb{N}_{0}^{d}$, we have

$$
\begin{aligned}
2^{-k_{1}-\cdots-k_{d}} \sum_{L \in \mathbb{N}_{0}\left(2^{K}\right)} W_{L}(X) & =\chi\left(\Delta_{K}^{0}, X\right), \\
2^{-k_{1}^{*}-\cdots-k_{d}^{*}} & \sum_{L \in \Pi_{K}} W_{L}(X)=R_{K}(X) \chi\left(\Delta_{K^{*}}^{0}, X\right) .
\end{aligned}
$$

The following simple facts concerning the elementary intervals (1.15) and boxes (1.16) will be useful in what follows. For each fixed $a \in \mathbb{N}_{0}$, the elementary intervals $\Delta_{a}^{m}$, $m \in \mathbb{N}_{0}\left(2^{a}\right)$, form a partition of the unit interval, and each point $x \in[0,1)$ belongs to exactly one of these intervals. If (1.6) is the dyadic representation of $x$, then $x \in \Delta_{a}^{m}$ with

$$
m=m(x)=\left[2^{a} x\right]=\sum_{i=1}^{a} 2^{a-i} \eta_{i}(x)=\sum_{i=1}^{a} \eta_{a+1-i}(x) 2^{i-1}
$$

here $[t]$ is the integral part of $t \in \mathbb{R}$, and formulas (2.44) determine the dyadic representation (2.1) for the number $m$.

Let $x, y \in[0,1)$, and let the sum $x \oplus y$ be well defined, say, $y \in \mathbb{Q}\left(2^{\infty}\right)$ (see $\left.\S 1\right)$. Using the dyadic representations of $x$ and $y$, we can check directly that $x \oplus y \in \Delta_{a}^{0}$ if and only if $m(x)=m(y)$, or in other words, $x$ and $y$ belong to the same elementary interval $\Delta_{a}^{m}$. This fact can be written as the following formula for the characteristic functions $\chi\left(\Delta_{a}^{m},.\right)$ of the intervals $\Delta_{a}^{m}$ :

$$
\chi\left(\Delta_{a}^{0}, x \oplus y\right)=\sum_{m \in \mathbb{N}_{0}\left(2^{a}\right)} \chi\left(\Delta_{a}^{m}, x\right) \chi\left(\Delta_{a}^{m}, y\right) .
$$

In the multidimensional case, this formula takes the form

$$
\chi\left(\Delta_{A}^{0}, X \oplus Y\right)=\sum_{M \in \mathbb{N}_{0}^{d}\left(2^{A}\right)} \chi\left(\Delta_{A}^{M}, X\right) \chi\left(\Delta_{A}^{M}, Y\right) .
$$

2.3. In the one-dimensional case, the Rademacher functions form a lacunary (in the Hadamard sense) subsystem of Walsh functions. This implies the following remarkable fact: if a function $f \in L_{2}(0,1)$ has a Fourier-Walsh series (2.19) containing only Rademacher functions,

$$
f(x) \simeq \sum_{\ell \geq 1} \widehat{f}_{\ell} r_{\ell}(x)
$$

then $f \in L_{q}(0, \infty)$ for all $q<\infty$, and all norms $\|f\|_{q}$ are equivalent to $\|f\|_{2}$. More precisely, the Khinchin inequality

$$
\beta_{q}\left(\sum_{\ell \geq 1} \widehat{f}_{\ell}^{2}\right)^{1 / 2} \leq\|f\|_{q} \leq \alpha_{q}\left(\sum_{\ell \geq 1} \widehat{f}_{\ell}^{2}\right)^{1 / 2}
$$

is valid with positive constants $\alpha_{q}$ and $\beta_{q}$ independent of $f$. In particular, one can take

$$
\alpha_{q}=\left(\frac{1}{2} q+1\right)^{1 / 2} \text {. }
$$

The Khinchin inequality (2.48) is among the basic facts of harmonic analysis and probability theory, its proof can be found in many sources (see, for example, [6, 13]). We 
refer also to the survey paper [9, where this inequality was discussed in a broad context, in particular, with attention to the exact values of the constants $\alpha_{q}$ and $\beta_{q}$.

In the present paper our concern is only with the right-hand inequality in (2.48) generalized to several variables.

Unfortunately, for $d>1$ the uniqueness of factorization of Walsh functions into the product of Rademacher functions is lost (compare with (2.27)); also, the $d$-dimensional Rademacher functions no longer form a subsystem lacunary in the Hadamard sense. Nevertheless, an analog of inequality (2.48) holds.

Using the right-hand inequality in (2.48) for $d=1$, together with induction on $d$, one can easily establish the following fact (see [13, Appendix D]): if a function $f \in L_{2}\left(U^{d}\right)$ has a Fourier-Walsh series (2.19) involving only the $d$-dimensional Rademacher functions,

$$
f(X) \simeq \sum_{L \in \mathbb{N}_{0}^{d}} \widehat{f}_{L} R_{L}(X),
$$

then $f \in L_{q}\left(U^{d}\right)$ for all $q<\infty$, and the $d$-dimensional Khinchin's inequality

$$
\|f\|_{q} \leq\left(2 \alpha_{q}\right)^{d}\left(\sum_{L \in \mathbb{N}_{0}^{d}} \widehat{f}_{L}^{2}\right)^{1 / 2}
$$

is valid, where $\alpha_{q}$ is the same constant as in (2.48).

Notice that (2.49) contains all Rademacher functions depending on $k \leq d$ variables; in particular, for $d=1$, in $(2.47)$ the constant term $\widehat{f}_{0} r_{0}(x)$ should be added. Hence, (2.50) involves the constant $2 \alpha_{q}$ instead of $\alpha_{q}$.

We shall need the following simple modification of inequality (2.50). Consider linear combinations of Rademacher functions

$$
\Omega_{K}(X)=\sum_{L \in \mathbb{N}_{0}^{d}} \Omega_{K, L} R_{L}(X), \quad K \in \mathbb{N}_{0}^{d},
$$

under the assumption that the coefficient matrix $\left(\Omega_{K, L}\right)$ generates a bounded operator $\Omega$ in the space $\ell_{2}\left(\mathbb{N}_{0}^{d}\right)$. Assume, for example, that the following conditions are fulfilled:

$$
\begin{aligned}
& \gamma_{1}=\sup _{K} \sum_{L}\left|\Omega_{K, L}\right|<\infty, \\
& \gamma_{2}=\sup _{L} \sum_{K}\left|\Omega_{K, L}\right|<\infty ;
\end{aligned}
$$

then the operator $\Omega$ is well known to be bounded, and $\|\Omega\| \leq \sqrt{\gamma_{1} \gamma_{2}}$.

With these remarks, inequality (2.50) immediately implies the following statement.

Lemma 2.3. Under conditions (2.52), for each $q<\infty$ and all square-summable sequences $\left\{b_{K}, K \in \mathbb{N}_{0}^{d}\right\}$ we have

$$
\left(\int_{U^{d}}\left|\sum_{K \in \mathbb{N}_{0}^{d}} b_{K} \Omega_{K}(Y)\right|^{q} d Y\right)^{1 / q} \leq\left[\gamma_{1} \gamma_{2}(q+2)\right]^{d / 2}\left(\sum_{K \in \mathbb{N}_{0}^{d}} b_{K}^{2}\right)^{1 / 2} .
$$

$\S 3$. Formulas FOR $L^{q}$-Discrepancies.

\section{DisCREPANCIES AND DISBALANCES OF DISTRIBUTIONS}

3.1. In order to obtain explicit formulas for the $L_{q}$-discrepancies $(1.2)$, we need the Fourier-Walsh expansion (2.19) for the characteristic functions $\chi\left(B_{Y}, \cdot\right)$ of the rectangular boxes $B_{Y}=[0, Y,) \times \cdots \times\left[0, Y_{d}\right) \subset U^{d}$ (see (1.15) and (1.16)). 
In the one-dimensional case, the characteristic function $\chi([0, y), \cdot)$ of an interval $[0, y) \subset$ $[0,1)$ has the Fourier-Walsh series

$$
\chi([0, y), x) \simeq \sum_{\ell \geq 0} \widehat{\chi}_{\ell}(y) w_{\ell}(x)
$$

with the Fourier-Walsh coefficients

$$
\widehat{\chi}_{\ell}(y)=\int_{0}^{y} w_{\ell}(x) d x .
$$

The evaluation of the integrals (3.2) is a good exercise with Walsh and Rademacher functions, but we prefer to refer to [5, §3], where this evaluation was already done. The answer is

$$
\widehat{\chi}_{\ell}(y)=2^{-\rho(\ell)} \psi_{\rho(\ell)}(y) w_{\ell}(y),
$$

where $\rho(\ell)$ is defined in (2.11), and the functions $\psi_{k}(y), k \geq 0$, are as follows:

$$
\psi_{0}(y)=\{y\}=\frac{1}{2}-\sum_{i \geq 1} 2^{-i-1} r_{i}(y)
$$

(see $(2.31))$, and, for $k \geq 1$,

$$
\psi_{k}(y)=\left\{2^{k-1} y+\frac{1}{2}\right\}-\frac{1}{2}=\frac{1}{2} r_{k}(y)+\left\{2^{k-1} y\right\}-\frac{1}{2}=\frac{1}{2} r_{k}(y)-\sum_{i \geq 1} 2^{-i-1} r_{k+i}(y),
$$

where $\{x\}$ is the fractional part of $x \in \mathbb{R}$.

A crucial point for our consideration is that the Fourier-Walsh series for functions $\psi_{k}(y)$ contain only Rademacher functions and, therefore, are lacunary.

These series converge absolutely and uniformly and satisfy

$$
\sup _{y}\left|\psi_{k}(y)\right| \leq 1
$$

Substituting (3.3) in (3.1), we obtain

$$
\chi([0, y), x) \simeq \sum_{\ell \geq 0} 2^{-\rho(\ell)} \psi_{\rho(\ell)}(y) w_{\ell}(y) w_{\ell}(x) .
$$

Here we are not yet able to use relation (2.16) to replace $w_{\ell}(y) w_{\ell}(x)$ by $w_{\ell}(y \oplus x)$, because $x$ and $y$ are arbitrary and, in general, the expression $y \oplus x$ is not well defined. In order to make such a transformation, we consider piecewise constant approximations $\chi^{(s)}([0, y), \cdot)$ to the characteristic functions $\chi([0, y), \cdot)$.

Using Lemma 2.1(i) and (3.5), we obtain

$$
\begin{aligned}
\chi^{(s)}([0, y), x) & =\sum_{\ell \in \mathbb{N}_{0}\left(2^{s}\right)} 2^{-\rho(\ell)} \psi_{\rho(l)}(y) w_{\ell}(y) w_{\ell}(x) \\
& =\sum_{\ell \in \mathbb{N}_{0}\left(2^{s}\right)} 2^{-\rho(\ell)} \psi_{\rho(\ell)}(y) w_{\ell}\left(y^{(s)} \oplus x^{(s)}\right),
\end{aligned}
$$

where the $y^{(s)}$ and $x^{(s)}$ are, respectively, the projections of $y$ and $x$ to $\mathbb{Q}\left(2^{s}\right)$ (see (1.9)).

We wish to estimate the accuracy of this approximation. Using (2.24) and (2.25), we obtain

$$
\chi([0, y), x)=x^{(s)}([0, y), x)+\theta(y, x) x\left(\Delta_{s}(y), x\right),
$$

where $\chi\left(\Delta_{s}(y), x\right)$ is the characteristic function of a unique elementary interval $\Delta_{s}(y)=$ $\left[m 2^{-s},(m+1) 2^{-s}\right), m=m(y)$, that includes the point $y$ (see $\left.(2.44)\right)$, and $\theta(y, x) \in[0,1)$ for all $y$ and $x$. Observe that

$$
0 \leq \chi^{(s)}([0, y), x) \leq 1 \quad \text { for all } y \text { and } x .
$$


We transform the sum (3.6) by grouping the terms with $\rho(\ell)=k$ together. By (2.36), we have

$$
\chi^{(s)}([0, y), x)=\sum_{k=0}^{s} 2^{-k} \psi_{k}(y) \sum_{\ell \in \Pi_{k}} w_{\ell}\left(y^{(s)} \oplus x^{(s)}\right) .
$$

The inner sum in (3.8) was calculated already in (2.38). Using (2.38), we obtain

$$
\begin{aligned}
\chi^{(s)}([0, y), x) & =\psi_{0}(y)+\sum_{k=1}^{s} 2^{-k} \frac{1}{2} \psi_{k}(y) r_{k}\left(y^{(s)} \oplus x^{(s)}\right) \chi\left(\Delta_{k-1}^{0}, y^{(s)} \oplus x^{(s)}\right) \\
& =\sum_{k=0}^{s} 2^{-k} \omega_{k}(y) r_{k}\left(y^{(s)} \oplus x^{(s)}\right) \chi\left(\Delta_{K^{*}}^{0}, y^{(s)} \oplus x^{(s)}\right),
\end{aligned}
$$

where, for convenience, we write

$$
\omega_{0}(y)=\psi_{0}(y), \quad \omega_{k}(y)=\frac{1}{2} \psi_{k}(y), \quad k \geq 1,
$$

and $K^{*}$ is defined as in $(2.39)$.

We write formula (3.9) for each coordinate axis of the unit cube, putting $y=y_{j}$ and $x=x_{j}, j=1, \ldots, d$, and multiply out these formulas. This results in the following piecewise constant approximation for the characteristic function of the box $B_{Y} \subset U^{d}$ :

$$
\chi^{(s)}\left(B_{y}, x\right)=\sum_{K \in[0, s]^{d}} 2^{-k_{1}-\cdots-k_{d}} \Omega_{K}(Y) R_{K}\left(Y^{(s)} \oplus X^{(s)}\right) \chi\left(\Delta_{K^{*}}^{0}, Y^{(s)} \oplus X^{(s)}\right) .
$$

Summation in $(3.11)$ is taken over all $K=\left(k_{1}, \ldots k_{j}\right)$ with $0 \leq k_{j} \leq s, j=1, \ldots, d$,

$$
\Omega_{K}(Y)=\prod_{j=1}^{d} \omega_{k_{j}}\left(y_{j}\right)
$$

$R_{K}(\cdot)$ is the $d$-dimensional Rademacher function $(2.28), K^{*}=\left(k_{1}^{*}, \ldots, k_{d}^{*}\right)$ with $k_{j}^{*}$ defined as in (2.39), $\Delta_{K^{*}}^{0} \subset U^{d}$ is the corresponding elementary box (1.16), and $Y^{(s)}$ and $X^{(s)}$ are the projections of the corresponding points $Y$ and $X$ to $\mathbb{Q}^{d}\left(2^{s}\right)$.

The following important property of the sum (3.11) should be mentioned (compare with Lemma 2.1(iii) and (2.13)):

$$
\chi^{(s)}\left(B_{Y}, X\right)=\chi^{(s)}\left(B_{Y}, X^{(s)}\right) .
$$

Notice also that the term with $K=0$ in the sum (3.11) is equal to $y_{1}, \ldots, y_{d}=\operatorname{vol} B_{Y}$.

Using (3.7), we can easily obtain the following bound for the accuracy of the approximation (3.11):

$$
\chi\left(B_{Y}, X\right)=\chi^{(s)}\left(B_{Y}, X\right)+\varepsilon^{(s)}(Y, X),
$$

where

$$
\varepsilon^{(s)}(Y, X)=\sum_{j=1}^{d} \theta_{j}(Y, X) \chi\left(\Delta_{s, j}(Y), X\right) ;
$$

here $\chi\left(\Delta_{s, j}(Y), X\right)$ is the characteristic function of the following elementary boxes:

$$
\Delta_{s, j}(Y)= \begin{cases}\Delta_{s}\left(y_{1}\right) \times[0,1)^{d-1} & \text { if } j=1, \\ {[0,1)^{j-1} \times \Delta_{s}\left(y_{j}\right) \times[0,1)^{d-j}} & \text { if } j=2, \ldots, d,\end{cases}
$$

and the $\theta_{j}(Y, X), j=1, \ldots, d$, are some functions satisfying $\left|\theta_{j}(Y, X)\right|<1$ for all $Y$ and $X$. 
Obviously, the sum (3.15) can be estimated as follows:

$$
\left|\varepsilon^{(s)}(Y, X)\right| \leq \sum_{j=1}^{d} \chi\left(\Delta_{s, j}(Y), X\right)
$$

3.2. Let $D \subset U^{d}$ be an arbitrary distribution of finitely many points. The local discrepancy $\mathcal{L}[D, Y]$ (see (1.1)) can be written in the form

$$
\mathcal{L}[D, Y]=\sum_{X \in D}\left(\chi\left(B_{Y}, X\right)-\operatorname{vol} B_{Y}\right) .
$$

Substituting the expression (3.14) in (3.18), we obtain

$$
\mathcal{L}[D, Y]=\mathcal{L}^{(s)}[D, Y]+\varepsilon^{(s)}[D, Y],
$$

where

and

$$
\mathcal{L}^{(s)}[D, Y]=\sum_{\chi \in D}\left(\chi^{(s)}\left(B_{Y}, x\right)-\operatorname{vol} B_{Y}\right)
$$

$$
\varepsilon^{(s)}[D, Y]=\sum_{\chi \in D} \varepsilon^{(s)}(Y, X)
$$

Using (3.11), we find

$$
\mathcal{L}^{(s)}[D, Y]=\sum_{K \in[0, s]^{d} \backslash\{0\}} 2^{-k_{1}-\cdots-k_{d}} \Omega_{K}(Y) \Phi_{K}\left[D, Y^{(s)}\right],
$$

where $K=\left(k, \ldots, k_{d}\right)$ and $\Phi_{K}\left[D, Y^{(s)}\right]$ looks like this:

$$
\Phi_{K}\left[D, Y^{(s)}\right]=\sum_{X \in D} R_{K}\left(Y^{(s)} \oplus X^{(s)}\right) \chi\left(\Delta_{K^{*}}^{0}, Y^{(s)} \oplus X^{(s)}\right) .
$$

Formulas (3.22) and (3.23) immediately imply the following important properties:

$$
\Phi_{K}\left[D, Y^{(s)}\right]=\Phi_{K}\left[D^{(s)}, Y^{(s)}\right], \quad \mathcal{L}^{(s)}[D, Y]=\mathcal{L}^{(s)}\left[D^{(s)}, Y\right],
$$

where

$$
D^{(s)}=\left\{X^{(s)}: X \in D\right\}
$$

is the projection of the distribution $D$ to $\mathbb{Q}^{d}\left(2^{s}\right)$.

Hence, the approximation (3.22) and the sums (3.23) depend only on the projection $D^{(s)}$ of the distribution $D$.

Using (3.15), we estimate (3.21):

$$
\left|\varepsilon^{(s)}[D, Y]\right|<\sum_{j=1}^{d} \sum_{X \in D} \chi\left(\Delta_{s, j}(Y), X\right)=\sum_{j=1}^{d} \#\left\{\Delta_{s, j}(Y) \cap D\right\} .
$$

We put

$$
\mu^{(s)}[D]=\max \left\{\#\left\{\Delta_{A}^{M} \cap D\right\}: \operatorname{vol} \Delta_{A}^{M}=2^{-s}\right\},
$$

where the maximum is calculated over all elementary boxes (1.16) of volume $\operatorname{vol} \Delta_{A}^{M}=$ $2^{-s}$. Since, for any $A \in \mathbb{N}_{0}\left(2^{s}\right)$, each shift $\Delta_{A}^{M} \oplus T=\Delta_{A}^{M(T)}$ is equivalent to a permutation of boxes $\Delta_{A}^{M}$, we have

$$
\mu^{(s)}[D \oplus T]=\mu[D], \quad T \in \mathbb{Q}^{d}\left(2^{s}\right) .
$$

From (3.26) and (3.27) we deduce the bound

$$
\sup _{Y}\left|\varepsilon^{(s)}[D, Y]\right|<d \mu^{(s)}[D] .
$$


Substituting (3.19) and (3.29) in (1.2) and using Minkowski's inequality, we obtain the following expression for the $L_{q}$-discrepancy:

$$
\mathcal{L}_{q}[D]=\mathcal{L}_{q}^{(s)}[D]+\theta d \mu[D],
$$

where

$$
\mathcal{L}_{q}^{(s)}[D]=\left(\int_{U^{d}}\left|\mathcal{L}^{(s)}[D, Y]\right|^{q} d Y\right)^{1 / q}
$$

and finally, for the mean values (1.14) we find

$$
\mathcal{M}_{s, q}[D]=\mathcal{M}_{s, q}^{(s)}[D]+\theta d \mu[D]
$$

where

$$
\mathcal{M}_{s, q}^{(s)}[D]=\left(2^{-d s} \sum_{T \in \mathbb{Q}^{d}\left(2^{s}\right)}\left(\mathcal{L}_{q}^{(s)}[D \oplus T]\right)^{q}\right)^{1 / q} .
$$

Note that $|\theta|<1$ in (3.30) and (3.32).

In view of (3.31), we can rewrite (3.33) as follows:

$$
\begin{aligned}
\left(\mathcal{M}_{s, q}^{(s)}[D]\right)^{q} & =2^{-d s} \sum_{T \in \mathbb{Q}^{d}\left(2^{s}\right)} \int_{U^{d}}\left|\mathcal{L}^{(s)}[D \oplus T, Y]\right|^{q} d Y \\
& =\int_{U^{d}} d Y 2^{-d s} \sum_{T \in \mathbb{Q}^{d}\left(2^{s}\right)}\left|\mathcal{L}^{(s)}[D \oplus T, Y]\right|^{q} .
\end{aligned}
$$

Consider the inner sum in (3.34). Using (3.22) and (3.23), we obtain

$$
\begin{aligned}
& \sum_{T \in \mathbb{Q}^{d}\left(2^{s}\right)}\left|\mathcal{L}^{(s)}[D \oplus T, Y]\right|^{q} \\
& =\sum_{T \in \mathbb{Q}^{d}\left(2^{s}\right)} \mid \sum_{K \neq 0} 2^{-k_{1}-\cdots-k_{j}} \Omega_{K}(Y) \\
& \quad \times\left.\sum_{X \in D} R_{K}\left(Y^{(s)} \oplus X^{(s)} \oplus T\right) \chi\left(\Delta_{K^{*}}^{0}, Y^{(s)} \oplus X^{(s)} \oplus T\right)\right|^{q} \\
& =\sum_{Z \in \mathbb{Q}^{d}\left(2^{s}\right)}\left|\sum_{K \neq 0} 2^{-k_{1}-\cdots-k_{j}} \Omega_{K}(Y) \sum_{X \in D} R_{K}\left(Z \oplus X^{(s)}\right) \chi\left(\Delta_{K^{*}}^{0}, Z \oplus X^{(s)}\right)\right|^{q},
\end{aligned}
$$

where, for each fixed $Y^{(s)} \in \mathbb{Q}^{d}\left(2^{s}\right)$, we have introduced a new variable $Z=Y^{(s)} \oplus T$.

Hence, the third sum in (3.35) is independent of $Y$. Substituting (3.35) in (3.34), we obtain

$$
\left.\mathcal{M}_{q}^{(s)}[D]\right)^{q}=2^{-d s} \sum_{Z \in \mathbb{Q}^{d}\left(2^{s}\right)} \int_{U^{d}}\left|\sum_{K \neq 0} \beta_{K}[D, Z] \Omega_{K}(Y)\right|^{q} d Y,
$$

where

$$
\beta_{K}[D, Z]=\sum_{X \in D} R_{K}\left(X^{(s)} \oplus Z\right) \chi\left(\Delta_{K^{*}}^{0}, X^{(s)} \oplus Z\right) .
$$

To simplify the notation for the sums over $K$ in (3.35) and (3.36), we do not indicate the summation domain

$$
K \in[0, s]^{d} \backslash\{0\} .
$$

Such a short notation will also be used in the formulas below. 
Using (2.16) and (2.46), we transform expression (3.37) as follows:

$$
\beta_{K}[D, Z]=\sum_{M \in \mathbb{N}_{0}^{d}\left(2^{K^{*}}\right)} R_{K}(Z) \chi\left(\Delta_{K^{*}}^{M}, Z\right) \sigma_{K, M}[D],
$$

where

$$
\sigma_{K, M}[D]=\sum_{X \in D} R_{K}(X) \chi\left(\Delta_{K^{*}}^{M}, X\right)
$$

This sum can be written as

$$
\sigma_{K, M}[D]=N_{K, M}^{+}[D]-N_{K, M}^{-}[D],
$$

where

$$
N_{K, M}^{ \pm}[D]=\#\left\{X \in D \cap \Delta_{K^{*}}^{M}: R_{K}(X)= \pm 1\right\} .
$$

For example, for $d=1$ and $k=1$, the only quantity (3.40) is $\sigma_{1,0}[D]=N_{1,0}^{+}-N_{1,0}^{-}$, where $N_{1,0}^{+}$and $N_{1,0}^{-}$are the numbers of points of $D$ belonging to the intervals $[0,1 / 2)$ and $[1 / 2,1)$, respectively. In connection with quadrature formulas, the quantities $(3.41)$ were discussed in 12 .

The quantity (3.40) should be called naturally the disbalance of a distribution $D$ in the elementary box $\Delta_{K^{*}}^{M}$.

Our nearest aim is to estimate the mean values (3.33) in terms of the disbalances (3.40).

For each $Z \in \mathbb{Q}^{d}\left(2^{s}\right)$, the integrals in (3.36) can be estimated with the help of the modified Khinchin's inequality from Lemma 2.1. For our specific system of functions (3.10), (3.12), the positive constants $\gamma_{1}, \gamma_{2}$ (see (2.52)) are estimated easily: $\gamma_{1}, \gamma_{2} \leq 1$. As a result, we obtain the bound

$$
\left(\mathcal{M}_{s, q}^{(s)}[D]\right)^{q} \leq(q+2)^{\frac{1}{2} d q} 2^{-d s} \sum_{Z \in \mathbb{Q}^{d}\left(2^{s}\right)}\left(\sum_{K \neq 0} \beta_{K}^{2}[D, Z]\right)^{q / 2}
$$

whence

$$
\left(\mathcal{M}_{s, q}^{(s)}[D]\right) \leq(q+2)^{d / 2}\left(2^{-d s} \sum_{Z \in \mathbb{Q}^{d}\left(2^{s}\right)}\left(\sum_{K \neq 0} \beta_{K}^{2}[D, Z]\right)^{q / 2}\right)^{1 / q} .
$$

Let $q \geq 2$. Applying Minkowski's inequality with the exponent $q / 2 \geq 1$ to the righthand side of (3.44), we obtain

$$
\left(M_{s, q}^{(s)}[D]\right) \leq(q+2)^{d / 2}\left(\sum_{K \neq 0}\left(2^{-d s} \sum_{Z \in \mathbb{Q}^{d}\left(2^{s}\right)}\left|\beta_{K}[D, Z]\right|^{q}\right)^{2 / q}\right)^{1 / 2} .
$$

For each $K$, we partition the unit cube to the following elementary boxes:

$$
U^{d}=\bigcup_{M \in \mathbb{N}_{0}^{d}\left(2^{K^{*}}\right)} \Delta_{K^{*}}^{M}
$$

Using this partition and formula (3.39), we see that

$$
\left|\beta_{K}[D, Z]\right|^{q}=\sum_{M \in \mathbb{N}_{0}^{d}\left(2^{K^{*}}\right)} \chi\left(\Delta_{K^{*}}^{M}, Z\right)\left|\sigma_{K, M}[D]\right|^{q} .
$$

It is easy to check that

$$
2^{-d s} \sum_{Z \in \mathbb{Q}^{d}\left(2^{s}\right)} \chi\left(\Delta_{K^{*}}^{M}, Z\right)=2^{-d s} \#\left\{\Delta_{K^{*}}^{M} \cap \mathbb{Q}^{d}\left(2^{s}\right)\right\}=\operatorname{vol} \Delta_{K^{*}}^{M}=2^{-k_{1}^{*}-\cdots-k_{d}^{*}}
$$

(for instance, it suffices to use Lemma 2.1(iii)). 
Substituting (3.47) and (3.48) in (3.44), we obtain

$$
\mathcal{M}_{s, q}^{(s)}[D] \leq(q+2)^{d / 2}\left(\sum_{K \neq 0} \tau_{q, K}^{2}[D]\right)^{1 / 2},
$$

where

$$
\tau_{q, K}[D]=\left(2^{-k^{*}-\cdots-k_{d}^{*}} \sum_{M \in \mathbb{N}_{0}^{d}\left(2^{K^{*}}\right)}\left|\sigma_{K, M}[D]\right|^{q}\right)^{1 / q} .
$$

The quantity (3.50) should be called naturally the $L_{q}$-mean disbalance on the partition (3.46).

We summarize the above discussion. Using formulas (3.32) and (3.49), we arrive at the following result.

Theorem 3.1. For all $q \geq 2$ and $s \in \mathbb{N}_{0}$, for any distribution $D \subset U^{d}$ the mean $L_{q}$-discrepancy (1.14) and the $L_{q}$-mean disbalances (3.50) are related by the inequality

$$
\mathcal{M}_{s, q}[D] \leq(q+2)^{\frac{1}{2} d}\left(\sum_{K \neq 0} \tau_{q, K}^{2}[D]\right)^{1 / 2}+d \mu^{(s)}[D],
$$

where $\mu^{(s)}[D]$ is the quantity defined in (3.27) and summation over $K$ in (3.51) is extended to the domain (3.38).

\section{§4. Proof of Theorem 1.1}

Theorem 3.1 was proved above for arbitrary distributions of finitely many points in the unit cube. To prove Theorem 1.1 it suffices to specialize a distribution $D$ in Theorem 3.1 as a $(\delta, s, d)$-net.

For $(\delta, s, d)$-nets, their disbalances (3.40) and (3.50) turn out to be small, and one should say that the $(\delta, s, d)$-nets are well-balanced distributions. In more precise terms this fact can be expressed as follows.

Lemma 4.1. For any $(\delta, s, d)$-net $D$ and any $q \geq 2$, the $L_{q}$-mean disbalances $(3.50)$ satisfy the relations

$$
\tau_{q, k}[D]=0
$$

if $0<k_{1}+\cdots+k_{d} \leq s-\delta$ and

$$
\tau_{q, k}[D] \leq 2^{\frac{1}{q}\left(d+s-k_{1}-\cdots-k_{d}\right)+\delta+d}
$$

if $k_{1}+\cdots+k_{d}>s-\delta$.

The proof of Lemma 3.1 is given below. Note that a certain improvement of (4.2) could be given:

$$
\tau_{q, k}[D] \leq 2^{\frac{1}{q}\left(d+s-k_{1}-\cdots-k_{d}\right)+\left(1-\frac{1}{q}\right) \delta+d},
$$

but even the bound (4.2) will suffice to prove Theorem 1.1.

Proof of Theorem 1.1. The definition of $(\delta, s, d)$-nets immediately implies the estimate

$$
\mu^{(s)}[D] \leq 2^{\delta},
$$

because each elementary box of volume $2^{-s}$ is contained in a larger elementary box of volume $2^{\delta-s}$ that contains exactly $2^{\delta}$ points of the net $D$.

Substituting (4.4) and relations (4.1) and (4.2) in inequality (3.51), we obtain

$$
\mathcal{M}_{s, q}[D] \leq(q+2)^{\frac{1}{2} d} 2^{\left(1+\frac{1}{q}\right)(d+\delta)}\left(\sum_{s, \delta}\right)^{\frac{1}{2}}+d 2^{\delta},
$$


where $\sum_{s, \delta}$ denotes the sum

$$
\sum_{s, \delta}=\sum_{k_{1}+\cdots+k_{d}>s-\delta} 2^{\frac{2}{q}\left(s-\delta-k_{1}-\cdots-k_{d}\right)}
$$

with summation over all $k=\left(k_{1}, \ldots, k_{d}\right) \in[0, s]^{d}$ such that $k_{1}+\cdots+k_{d}>s-\delta$.

The sum (4.6) can be written in the form

$$
\sum_{s, \delta}=\sum_{t=1}^{d s} a(t) 2^{-\frac{2}{q} t}
$$

where

$$
a(t)=\#\left\{K \in[0, s]^{d}: k_{1}+\cdots+k_{d}=t\right\} .
$$

It is easily seen that $a(t) \leq(s+1)^{d-1}$ for all $t$. Therefore,

$$
\sum_{s, \delta}<(s+1)^{d-1} \sum_{t=1}^{\infty} 2^{-\frac{2}{q} t}=(s+1)^{d-1} 2^{-\frac{2}{q}}\left(1-2^{-\frac{2}{q}}\right)^{-1}<q(s+1)^{d-1} .
$$

Substituting (4.8) in (4.5) and recalling that $q \geq 2$, we get

$$
\mathcal{M}_{s, q}[D]<2^{2 d+2 \delta+2} q^{\frac{1}{2}(d+1)}(s+1)^{\frac{1}{2}(d-1)} .
$$

The proof of Theorem 1.1 is complete.

Proof of Lemma 4.1. Identity (4.1) follows from the definitions of the $(\delta, s, d)$-nets and the disbalances (3.40), (3.50). Indeed, if $K \neq 0$, then an elementary box $\Delta_{K^{*}}^{M}$ of volume $2^{-k_{1}^{*}-\cdots-k_{j}^{*}}$ can be split into an even number $2^{\varkappa(K)}$ of smaller elementary boxes $\Delta_{K}^{M^{\prime}}$ of volume $2^{-k_{1}-\cdots-k_{d}}$. Here $\varkappa(K)$ is equal to the number of nonzero components of the vector $K=\left(k_{1}, \ldots, k_{d}\right)$; obviously, we have $1 \leq \varkappa(K) \leq d, K \neq 0$, and

$$
k_{1}^{*}+\cdots+k_{d}^{*}=k_{1}+\cdots+k_{d}-\varkappa(K) .
$$

On one half of these smaller boxes the Rademacher function $R_{K}(X)$ is equal to 1 , and on the other half, $R_{K}(X)=-1$. If $k_{1}+\cdots+k_{d} \leq s-\delta$, then $2^{-k_{1}-\cdots-k_{d}} \geq 2^{\delta-s}$, and this means that each of the boxes $\Delta_{K}^{M_{1}}$ can be split into $2^{s-\delta-k_{1}-\cdots-k_{d}}$ yet other smaller boxes of volume $2^{\delta-s}$, and by the definition of $(\delta, s, d)$-nets, each of these smaller boxes contains exactly $2^{\delta}$ points of the distribution $D$. Hence, by (3.42), we obtain

$$
N_{K, M}^{+}[D]=N_{K, M}^{-}[D]=2^{\delta} \cdot 2^{s-\delta-k_{1}-\cdots-k_{d}} \cdot 2^{\varkappa(K)-1}=2^{s-k_{1}^{*}-\cdots-k^{*}-1} .
$$

Therefore, $\sigma_{K, M}[D]=0$ and $\tau_{q, K}[D]=0$.

The proof of (4.1) is complete.

Now we prove inequalities (4.2). Let $k_{1}+\cdots+k_{d}>s-\delta$. Then the volume of any elementary box $\Delta_{K}^{M}$ is less than $2^{\delta-s}$, and the definition of the $(\delta, s, d)$-nets implies $\#\left\{D \cap \Delta_{K}^{M}\right\} \leq 2^{\delta}$. On the other hand, each elementary box $\Delta_{K^{*}}^{M}$ can be split into $2^{\varkappa(K)} \leq 2^{d}$ smaller boxes $\Delta_{K}^{M^{\prime}}$, whence $\#\left\{D \cap \Delta_{K^{*}}^{M}\right\} \leq 2^{d+\delta}$. With this bound, the disbalance (3.40) can be estimated as follows:

$$
\left|\sigma_{K, M}[D]\right| \leq \#\left\{D \cap \Delta_{K^{*}}^{M}\right\} \varepsilon_{K^{*}, M} \leq 2^{d+\delta} \varepsilon_{K^{*}, M},
$$

where

$$
\varepsilon_{K^{*}, M}= \begin{cases}0 & \text { if } D \cap \Delta_{K^{*}}^{M}=\varnothing \\ 1 & \text { if } D \cap \Delta_{K^{*}}^{M} \neq \varnothing\end{cases}
$$

Observe that

$$
\sum_{M \in \mathbb{N}_{0}^{d}\left(2^{K^{*}}\right)} \varepsilon_{K^{*}, M} \leq \#\{D\}=2^{s}
$$


Substituting (4.11) in formula (3.50) for the disbalance and taking (4.13) and (4.10) into account, we obtain

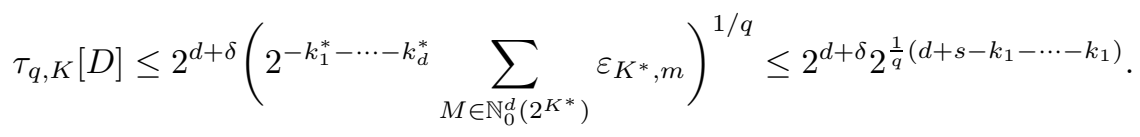

This completes the proof of inequalities (4.2).

Thus, Lemma 4.1 is proved.

\section{REFERENCES}

[1] J. Beck and W. W. L. Chen, Irregularities of distributions, Cambridge Tracts in Math., vol. 89, Cambridge Univ. Press, Cambridge, 1987. MR0903025 (88m:11061)

[2] W. W. L. Chen, On irregularities of distribution. II, Quart. J. Math. Oxford Ser. 234 (1983), no. 135, 257-279. MR0711520 (85c:11065)

[3] W. W. L. Chen and M. M. Skriganov, Orthogonality and digit shifts in the classical mean squares problem in irregularities of point distribution, Diophantine Approximation, Festschrift dedicated to Wolfgang Schmidt's 70th birthday (H. P. Schlickewei et al., eds.), Dev. Math., vol. 16, SpringerVerlag, Berlin, 2008, pp. 141-159. MR2487691 (2009m:11119)

[4] H. Faure, Discrépance de suites associées à un système de numération (en dimension s), Acta Arith. 41 (1982), 337-351. MR0677547 (84m:10050)

[5] N. J. Fine, On the Walsh functions, Trans. Amer. Math. Soc. 65 (1949), 372-414. MR0032833 $(11: 352 \mathrm{~b})$

[6] B. I. Golubov, A. V. Efimov, and V. A. Skvortsov, Walsh series and transforms. Theory and applications, Nauka, Moscow, 1987; English transl., Math. Appl. (Soviet Ser.), vol. 64, Kluwer Acad. Publ. Group, Dordrecht, 1991. MR0925004 (89h:42031). MR.1155844 (92m:42031)

[7] J. Matoušek, Geometric discrepancy. An illustrated guide, Algorithms Combin., vol. 18, SpringerVerlag, Berlin, 1999. MR,1697825(2001a:11135)

[8] H. Niederreiter, Nets, $(t, s)$-sequences, and algebraic curves over finite fields with many rational points, Internat. Congress of Mathematicians. Vol. III (Berlin, 1998), Doc. Math. 1998, Extra Vol. III, 377-386. MR1648171 (99k:11090)

[9] G. Peshkir and A. N. Shiryaev, Khinchin inequalities and a martingale extension of the sphere of their action, Uspekhi Mat. Nauk 50 (1995), no. 5, 3-62; English transl., Russian Math. Surveys 50 (1995), no. 5, 849-904. MR1365047 (96k:60038)

[10] M. M. Skriganov, Coding theory and uniform distributions, Algebra i Analiz 13 (2001), no. 2, 191239; English transl., St. Petersburg Math. J. 13 (2002), no. 2, 301-337. MR1834866 (2002g:11111)

[11] Harmonic analysis on totally disconnected groups and irregularities of point distributions, J. Reine Angew. Math. 600 (2006), 25-49. MR2283797(2007k:11122)

[12] I. M. Sobol', Multidimensional quadrature formulae and Haar functions, Nauka, Moscow, 1969. (Russian) MR 0422968 (54:10952)

[13] E. M. Stein, Singular integrals and differentiability properties of functions, Princeton Math. Ser., No. 30, Princeton Univ. Press, Princeton, NJ, 1970. MR0290095 (44:7280)

St. Petersburg Branch, Steklov Mathematical Institute, Russian Academy of Sciences, Fontanka 27, St. Petersburg 191023, Russia

E-mail address: skrig@pdmi.ras.ru

Received 27/JAN/2011

Translated by THE AUTHOR 